\title{
Does Concurrent Existence of Asthma or COPD Affect Susceptibility \& Outcomes to Novel Coronavirus Infections?
}

\section{A Short Review of Evidence and Mechanisms}

\begin{abstract}
The SARS-CoV-2 virus causing COVID-19 pandemic across the world has highlighted the risk of human-to-human transmission, the speed of spread in a globally mobile population and the need for health services to be flexible in their responses to hitherto unknown challenges. One of the hallmarks of COVID-19 is severe acute respiratory syndrome presenting with widespread viral pneumonitis and respiratory failure. It is therefore not unusual for patients with chronic lung diseases such as asthma, COPD and bronchiectasis to be concerned regarding their particular vulnerability. As healthcare professionals responsible for the care of such patients, it is important to understand how the SARS-CoV-2 virus impacts on the respiratory system and the outcomes for patients with chronic lung disease. This review examines the evidence and offers sensible guidance until more is known.
\end{abstract}

\section{Keywords}

Asthma, COPD, COVID19, ACE2 receptors

\section{Background}

As the world faces a pandemic with the novel coronavirus "severe acute respiratory syndrome coronavirus 2" (SARSCoV-2) causing a spectrum of rapidly spreading illness dubbed COVID-19, one of the key anxieties relates to the susceptibility and outcomes amongst patients with preexisting lung disease. The typical patients with symptomatic infection present with fever, cough, breathlessness and gastrointestinal symptoms. Profound hypoxia appears to be a typical picture and often patients progress to needing early invasive mechanical ventilation rapidly. The radiological picture is one of bilateral infiltrates typically seen in adult respiratory distress syndrome (ARDS), the full blood count shows lymphopaenia while the inflammatory markers including non-specific C-reactive protein, $d$-Dimer and procalcitonin are elevated. There is very little data available currently from

the

\author{
Akash Srinivasan ${ }^{1}$, Indranil Chakravorty PhD \\ $\mathrm{FRCP}^{2}$, Koottalai Srinivasan MD FRCP ${ }^{3}$ \\ 1 Imperial College Medical School, London \\ 2 St George's University of London, London \\ 3 Keele University Medical School, Princess \\ Royal Hospital, Telford \\ k.srinivasan@btinternet.com
}

cite as: Srinivasan, A., Chakravorty, I., Srinivasan, K. (2020) Does concurrent existence of asthma or COPD affect susceptibility \& outcomes to novel coronavirus infections? A short review of evidence and mechanisms. The Physician vol 6: issue 2: 1-9 DOI: https://doi.org/10.38192/1.6.1.2

\begin{tabular}{ll}
\multicolumn{2}{l}{ Article Information } \\
Submitted & 01.05 .2020 \\
ePub & 06.04 .2020 \\
Revised & 18.11 .2020 \\
Published22.11.2020
\end{tabular}

Creative Commons Licence v4.0

analysis of outbreaks in China and South Korea, but patients and physicians are faced with the challenge of giving common sense but evidence-based guidance. The mortality appears to be variable and is reported between 3-10\%. Particularly vulnerable are the elderly and those with hypertension, diabetes or kidney disease. ${ }^{1}$ There is a presumption that patients with respiratory disease will have negative outcomes when infected with coronavirus. However, the evidence suggests that this may not be the case. This article will attempt to explore the vulnerabilities 
and draw a quick set of conclusions until more information is available.

\section{Virology}

Human coronaviruses are common throughout the world. Coronaviruses are enveloped, non-segmented, singlestranded, positive-sense ribonucleic acid (RNA) viruses named after their corona- or crown-like surface projections observed on electron microscopy that correspond to large surface spike proteins. These viruses are host-specific and can infect humans and a variety of different animals as well. Four distinct genera have been identified: alpha-, beta- gamma-, and delta-coronavirus. SARS and middle eastern respiratory syndrome (MERS) are both in the genus beta-coronavirus. ${ }^{2}$ Based on the preliminary information of this novel virus, it is considered that SARS-CoV-2 is the third zoonotic human coronavirus of the century and is transmissible from person to person. Currently, it is still unclear about the origins and possible intermediate animal vectors of SARS-CoV-2 although, in addition to bats, the pangolin species has been highlighted as a natural reservoir of similar coronaviruses, based on analysis at a whole-genome level. ${ }^{3}$ The exact mechanism of human-to-human transmission is also unclear but it is thought to occur through the inhalation of respiratory droplets or coming in contact with infected surfaces prior to touching the eyes, nose or mouth. ${ }^{4}$

\section{Respiratory Diseases}

It is well established that respiratory tract viral infections are associated with asthma exacerbations. Several types of viruses, such as rhinovirus (RVs) and respiratory syncytial virus (RSV) have been implicated, whereas for coronavirus there is no data yet. There is consensus that a hypersensitivity state, a genetic predisposition, deficient antiviral response, impairment of immune cell function, damage of epithelium, cytokine and chemokine response may all contribute to the synergistic promotion of allergic disease exacerbation by allergen and virus, especially for respiratory tract allergy. ${ }^{5,6}$

The prevalence of chronic obstructive pulmonary disease (COPD) in people $\geq 40$ years old was $13.7 \%$ in China and respiratory tract virus infection is a common trigger for acute exacerbations. ${ }^{7}$ However, the prevalence of SARSCoV-2 infection in COPD patients is not clear and the influence of smoking behaviour on the susceptibility to this virus has not been investigated. Huang et al. ${ }^{8,9}$ have shown a rate of chronic COPD of approximately $2 \%$ and $2.9 \%$, respectively amongst COVID-19 patients in Wuhan. ${ }^{10}$ Subsequently, Chen et al. 10 have reported a rate of approximately $1 \%$ for respiratory system diseases, different from an estimated COPD prevalence of between $1.2-8.9 \%$ in different regions of China. Moreover, interstitial lung disease, bronchiectasis or asthma appear to be under-reported. ${ }^{8}$

In previous coronavirus epidemics, lower respiratory tract infections (LRTI)s with coronavirus in infants and children tended to be mild, unless the infant or child has an infection with a co-viral pathogen, has an underlying pulmonary disorder like asthma or cystic fibrosis, or is immunocompromised (haematological malignancy or solid tumour). ${ }^{2}$

\section{Mechanism}

Majority of patients with acute wheezy respiratory illness, (62\%-95\% of children and $41-78 \%$ of adults) have demonstrable infection with respiratory viruses in both hospital and community settings. Rhinoviruses (RVs) are the most frequently detected type of virus at all ages, whose infection could lead to more severe and longerlasting lower respiratory tract symptoms. In addition to RV, other respiratory tract viruses, such as respiratory syncytial virus (RSV), influenza viruses (IfVs), coronaviruses (CoVs), human metapneumoviruses (HMPVs), parainfluenza viruses (PIVs), adenoviruses (AdVs), and bocaviruses (BoVs), had all been detected in subjects with asthma exacerbations. $^{7}$

Coronaviruses such as CoV-OC43 and CoV-229E are transmitted primarily during winter have been loosely linked to asthma exacerbations in children and adults. However, in a systematic review the pooled prevalence of CoV infection during asthma exacerbations was only $8 \%$, ranking below RV (42.1\%, the highest), RSV, herpes simplex virus (HSV), enterovirus (EnV), and IfV. Overall, CoV seemed to have a minor, if any, contribution to acute asthma exacerbations, and CoV infections were frequently co-infected with other viruses.

Most animal models found no definite association with SARS-CoV and MERS-CoV which could cause severe respiratory disease in humans, and asthma. However, in a retrospective study of children admitted to hospital in Seattle with LRTI with coronavirus, the children who were immunocompromised or were infected by a co-pathogen, i.e RSV or had an underlying pulmonary disorder, were at greater risk for severe LRTI. ${ }^{9}$

Patients experiencing exacerbations of severe COPD usually demonstrate existence of multiple viruses concurrently in their airways suggesting that host response to viral infections may be dysregulated in COPD. ${ }^{11,12}$ Human CoV, in particular HCoV-229E, HCoV-OC43, HCoVNL63, and HCoV-HKU1, were shown to trigger acute respiratory illness in elderly adults with COPD and can result in hospitalisation. ${ }^{13}$ Activation of the innate immune axis leads to increased numbers of invariant natural killer T-cells (iNKT-cells) and alternatively activated interleukin 
IL-13-producing macrophages in the lung. Thus, viruses cause a more severe disease exacerbation with a heightened inflammatory response leading to an accelerated loss of lung function. ${ }^{14}$

\section{Infection Pathways}

The epithelial cell is the principal site of viral infection in the airways and plays a central role in viral modulation of airway inflammation by the release of a variety of cytokines, chemokines, and growth factors. Antiviral defence depends at least in part on a network of mucosal epithelial cells and macrophages. When this network is compromised, the host is highly susceptible to infection, but network components can be engineered to provide increased resistance. Development of in vitro experimental models of virus infection has identified interferon IFN- $\beta$ and nitric oxide (NO) as possible therapeutic targets to augment antiviral immunity, and (nuclear factor kappalight-chain-

enhancer of activated $B$ cells) NFKB as a target for the development of anti-inflammatory therapies. ${ }^{15}$

Viral entry relies on a fine interplay between the virion and the host cell. Infection is initiated by the interaction of the viral particle with specific proteins on the cell surface. After initial binding of the receptor, enveloped viruses need to fuse their envelope with the host cell membrane to deliver their nucleocapsid to the target cell. Coronaviruses use a variety of receptors and triggers to activate fusion, however fundamental aspects that enable this initial step of the viral life cycle to be conserved. ${ }^{16}$ The receptor of the SARS-CoV is the angiotensin-converting enzyme 2 (ACE2). ${ }^{17}$
ACE2 is a type I integral membrane protein abundantly expressed in lung and gastric tissue; it is a monocarboxypeptidase that hydrolyses angiotensin II. There is experimental evidence of the likelihood of genetic overexpression or pharmacological activation of the renin angiotensin system by upregulation of the ACE 2 in diabetic $\mathrm{db} / \mathrm{db}$ mice. ${ }^{18}$ There is an emerging hypothesis therefore that this upregulation of the RAS may play a role in the observed increased susceptibility of patients with hypertension or cardiovascular disease to coronaviruses. Furthermore, due to the distribution of ACE2 in the body, the lung is one of the main viral entry points.

\section{Transmission by Aerosol}

Aerosol-generating procedures (AGPs) are increasingly recognised as important sources for nosocomial transmission of viruses such as MERS-CoV and SARS-CoV, for example, intubation was investigated as a possible cause of Ebola virus (EBoV) transmission among healthcare workers in the United States. ${ }^{19,20}$ With regards to COVID-19, a study looking at the stability of SARS-CoV-2 found that aerosol and fomite transmission of the virus is plausible because the virus appears to stay viable and infectious in aerosols for hours. ${ }^{21}$ The site of deposition after an inhalational event can affect disease kinetics and pathogenesis, however, the deposition of respiratory pathogens in the lungs will generally result in the more rapid aggressive infection with higher mortality rates. Evidence exists in both animal models and humans for a number of entry/ deposition sites include upper airway lymphoid tissues. lower respiratory tract, the olfactory system, GI tract, and conjunctiva. ${ }^{22}$

Table 1: Potential aerosol-generating medical procedures involved in nosocomial virus transmission. (AGMP How/Where Aerosols May Be Generated)

Bronchoscopy * Induced cough, respiratory tract

Cardiopulmonary resuscitation * Induced cough, respiratory tract

Non-invasive ventilation *

(BiPAP, CPAP, HFOV) Possible mechanical dispersal of aerosols,

Tracheal intubation * respiratory tract

Manual ventilation *

Induced cough, respiratory tract

Surgery

Sputum induction -

Nebuliser treatment -

Suctioning -

Laser plume -

Possible mechanical dispersal of aerosols, respiratory tract

Cutting bone and tendon, and irrigation aerosolise blood

Induced cough, respiratory tract

Possible mechanical dispersal of aerosols, respiratory tract

Possible mechanical dispersal of aerosols, respiratory tract

Mechanical dispersal of aerosols

* Possible association with SARs-CoV transmission ${ }^{23}$

In a meta-analysis, procedures reported to present an increased risk of transmission included tracheal intubation odds ratio 6.6 (confidence interval $\mathrm{Cl}$ 2.3, 18.9), tracheostomy [OR $4.2(\mathrm{Cl} 1.5,11.5)]$, non-invasive ventilation [OR $3.1(1.4,6.8)]$, manual ventilation before 
intubation [OR $2.8(\mathrm{Cl} 1.3,6.4)$ ] and unprotected eye contact with secretions $(O R=7.34, p=.001) .^{20,24}$

Other intubation associated procedures, endotracheal aspiration, suction of body fluids, bronchoscopy, nebuliser treatment, administration of $\mathrm{O} 2$, high flow $\mathrm{O} 2$, manipulation of $\mathrm{O} 2$ mask or BiPAP mask, defibrillation, chest compressions, insertion of a nasogastric tube, and collection of sputum were not statistically significant. ${ }^{23,24}$ Theoretically nebulisation also generates droplets and if nebulisation is associated with a productive cough this may expose staff or bystanders by the airborne route to nosocomial infection which is a widely held expert opinion though still remains controversial. ${ }^{25,26}$ Use of highfrequency oscillation, high-flow nasal oxygen and continuous positive airway pressure are also highly likely to pose a risk of transmission to healthcare staff. Otolaryngological and dental procedures utilising highfrequency mechanical devices also pose a significant risk. However, there is a scientific debate on how evidence from such studies should be interpreted into national personal protection guidance for frontline healthcare staff. It is Public Health England's view that administering pressurised humidified oxygen and nebulised medication are not thought to carry a significant infection risk because the aerosol is generated from material other than the patient's secretions. $^{27}$

Investigation of small cohorts of nosocomial infection in 2003, showed that staff who used masks, gowns, and handwashing were less likely to develop SARS than those who did not use them, but the association for gloves was not significant. Both surgical and US National Institute for Occupational Safety and Health (N95) masks were significantly associated with protection. The data suggested that precautions against droplets and contact may be adequate for prevention of nosocomial SARS, where no AGPs are undertaken. ${ }^{28}$

\section{Treatment options}

Currently, the mainstay of response against the COVID-19 pandemic is based on avoidance of transmission, particularly to the vulnerable populations, social distancing measures, personal protection and hand hygiene. ${ }^{29} \mathrm{WHO}$ recommends strategies for extensive tracing - testing of all contacts and when community transmission is established moving to a strategy of comprehensive detection by widespread testing. ${ }^{30}$

For patients presenting with SARS to hospital, there is a need for the rapid expansion of intensive care capacity as this may determine mortality. Supportive care - including management of organ failure and prevention of complications, especially acute respiratory distress syndrome, organ failure and secondary nosocomial infections - remains the most important management strategy for SARS and MERS, as there is currently no specific antiviral treatment that has been proven to be effective in randomised controlled trials. There are no established treatment regimens, but rapid, open-label trials are underway.

The approach to treatment is based on;

- Virus-based therapies include monoclonal antibodies and antiviral peptides that target the viral spike glycoprotein, viral enzyme inhibitors, viral nucleic acid synthesis inhibitors and inhibitors of other viral structural and accessory proteins.

- Host-based therapies include agents that potentiate the interferon response or affect either host signalling pathways involved in viral replication or host factors utilised by coronaviruses for viral replication. ${ }^{31}$

\section{Inhaled corticosteroids}

The inclusion of inhaled corticosteroids in the FDArequested label change warning physicians that treatment with corticosteroids may place patients with viral infections at increased risk of serious complications does not appear to be warranted. Steroids have been used by some Chinese physicians in the treatment of COVID-19 and it is thought to able to stop the cytokine storm; however, this only appears to occur during a narrow window after the SARS-CoV-2 virus has been eliminated by the human immune response because otherwise, the virus could replicate faster and exacerbate symptoms. ${ }^{32}$ It is also hypothesised that routine use of corticosteroids to treat this virus could result in the exacerbation of COVID-19related lung injury and in cases of ARDS resulting from COVID-19, it has been suggested that glucocorticoids should be avoided based on the evidence that they can be harmful in viral pneumonia and ARDS caused by influenza. ${ }^{33,34,35}$

However, so far there does not appear to be any evidence linking the daily use of corticosteroid inhalers as a preventer of asthma attacks to poorer outcomes once the patient contracts COVID-19.

Ernst and co-workers used a large health database to identify patients with COPD and to study the frequency of inhaled corticosteroid use in those admitted to hospital because of pneumonia compared with matched control subjects. Current use of inhaled corticosteroids was associated with an increase of $70 \%$ in the rate of hospital admission for pneumonia. The admission rate was greatest with the highest doses of corticosteroids used, and a reduction in risk once inhaled corticosteroids were stopped was observed. A $53 \%$ increase in pneumonia death within 30 days of admission with inhaled corticosteroids was also found. ${ }^{36}$ This has also been shown in large epidemiological association in South Korea with 
asthma patients. ${ }^{37}$ However in a cohort of viral pneumonia patients the inhaled corticosteroid (fluticasone) was associated with a lower risk of pulmonary infiltrates in patients with influenza $A(H 1 N 1)$ infection. ${ }^{38}$

\section{Mortality}

The Intensive Care National Critical Care Audit Centre (ICNARC) data from approximately 2,500 admissions to intensive care units in the UK published on 4 April, shows only $1.1 \%$ of patients admitted to intensive care units and $1.3 \%$ needing advanced ventilatory support had underlying respiratory disease. ${ }^{39}$

\section{Conclusion}

SARS-Cov-2 is a severe threat to a huge swathe of the world population. Patients who are older, with co-morbidities such as hypertension, diabetes, kidney disease remain at significantly higher risk of poor outcomes. It is understandable that patients and health care workers with asthma or COPD are likely to be concerned with the potential for being severely affected. However, the data from China and the UK does not indicate that patients with underlying COPD or asthma have an increased incidence of coronavirus infection or are at risk of significant adverse outcomes.

It is well established that these patients are at greater risk of viral infections (not coronavirus) and combination of viruses or secondary bacterial infections are associated with LRTIs and need for hospital treatment. There is a small but real risk of nosocomial infections and transmission by nebuliser therapy of viral fomites/droplets, although the UK PHE guidance does not classify this as such.

Hence, until further robust data is available, here are some common-sense tips;

- Recommend practice of strict transmission avoidance and social distancing

- Recommend vigilance in personal protection by frequent hand washing or use of a surgical mask (against droplet transmission)

- Recommend the wearing of face coverings in public to reduce inadvertent asymptomatic transmission to others

- Monitor asthma control and if possible recommend a lower dose of ICS

- Avoid nebuliser use

- If there are signs of exacerbation, treat promptly avoiding prolonged or high dose corticosteroid use and consider early treatment of secondary bacterial infections

- If admitted, consider entry into trials of antivirals, chloroquine or combinations

\section{REFERENCES}

1. Zhang, J. et al. Clinical characteristics of 140 patients infected with SARS-CoV-2 in Wuhan, China. Allergy https://onlinelibrary.wiley.com/doi/abs/10.1111/all.1423 8 (2020).

2. Hageman, J. R. The Coronavirus Disease 2019 (COVID19). Pediatr. Ann. 49, e99-e100 (2020).

3. Zhang, T., Wu, Q. \& Zhang, Z. Probable Pangolin Origin of SARS-CoV-2 Associated with the COVID-19 Outbreak. Curr. Biol. 0, (2020).

4. Qu, G., Li, X., Hu, L. \& Jiang, G. An Imperative Need for Research on the Role of Environmental Factors in Transmission of Novel Coronavirus (COVID-19). Environ. Sci. Technol. (2020) doi:10.1021/acs.est.0c01102.

5. Wu, Y.-H. et al. Pulmonary IL-33 orchestrates innate immune cells to mediate RSV-evoked airway hyperreactivity and eosinophilia. Allergy n/a,.

6. Gavala, M. L., Bashir, H. \& Gern, J. E. Virus/Allergen Interactions in Asthma. Curr. Allergy Asthma Rep. 13, 298307 (2013).

7. Kurai, D., Saraya, T., Ishii, H. \& Takizawa, H. Virusinduced exacerbations in asthma and COPD. Front. Microbiol. 4, (2013).

8. Lupia, T. et al. 2019 novel coronavirus (2019-nCoV) outbreak: A new challenge. J. Glob. Antimicrob. Resist. 21, 22-27 (2020).

9. Huang, C. et al. Clinical features of patients infected with 2019 novel coronavirus in Wuhan, China. The Lancet 395, 497-506 (2020).

10. Chen, N. et al. Epidemiological and clinical characteristics of 99 cases of 2019 novel coronavirus pneumonia in Wuhan, China: a descriptive study. The Lancet 395, 507-513 (2020).

11. McManus, T. E. et al. Respiratory viral infection in exacerbations of COPD. Respir. Med. 102, 1575-1580 (2008).

12. Monto, A. S. Epidemiology of Respiratory Viruses in Persons with and without Asthma and COPD. Am. J. Respir. Crit. Care Med. 151, 1653-1658 (1995).

13. Gorse, G. J., O’Connor, T. Z., Hall, S. L., Vitale, J. N. \& Nichol, K. L. Human Coronavirus and Acute Respiratory Illness in Older Adults with Chronic Obstructive Pulmonary Disease. J. Infect. Dis. 199, 847-857 (2009).

14. Frickmann, $H$. et al. The influence of virus infections on the course of COPD. Eur. J. Microbiol. Immunol. 2, 176-185 (2012).

15. Johnston, S. L. Overview of Virus-induced Airway Disease. Proc. Am. Thorac. Soc. 2, 150-156 (2005).

16. Belouzard, S., Millet, J. K., Licitra, B. N. \& Whittaker, G. R. Mechanisms of coronavirus cell entry mediated by the viral spike protein. Viruses 4, 1011-1033 (2012).

17. Li, W. et al. Angiotensin-converting enzyme 2 is a functional receptor for the SARS coronavirus. Nature 426 , 450-454 (2003).

18. Zhang, Y. et al. Upregulation of Angiotensin (1-7)Mediated Signaling Preserves Endothelial Function 
Through Reducing Oxidative Stress in Diabetes. Antioxid. Redox Signal. 23, 880-892 (2015).

19. McCarthy, M. Texas healthcare worker is diagnosed with Ebola. BMJ 349, (2014).

20. Judson, S. D. \& Munster, V. J. Nosocomial Transmission of Emerging Viruses via Aerosol-Generating Medical Procedures. Viruses 11, (2019).

21. van Doremalen, N. et al. Aerosol and Surface Stability of SARS-CoV-2 as Compared with SARS-CoV-1. N. Engl. J. Med. 0, null (2020).

22. Thomas, R. J. Particle size and pathogenicity in the respiratory tract. Virulence 4, 847-858 (2013).

23. Tran, K., Cimon, K., Severn, M., Pessoa-Silva, C. L. \& Conly, J. Aerosol generating procedures and risk of transmission of acute respiratory infections to healthcare workers: a systematic review. PloS One 7, e35797 (2012).

24. Raboud, J. et al. Risk factors for SARS transmission from patients requiring intubation: a multicentre investigation in Toronto, Canada. PloS One 5, e10717 (2010).

25. Wan, G.-H., Tsai, Y.-H., Wu, Y.-K. \& Tsao, K.-C. A largevolume nebulizer would not be an infectious source for severe acute respiratory syndrome. Infect. Control Hosp. Epidemiol. 25, 1113-1115 (2004).

26. Booth, T. F. et al. Detection of airborne severe acute respiratory syndrome (SARS) coronavirus and environmental contamination in SARS outbreak units. J. Infect. Dis. 191, 1472-1477 (2005).

27. Public Health England. Guidance COVID-19 personal protective equipment (PPE). Available from: https://www.gov.uk/government/publications/wuhannovel-coronavirus-infection-prevention-andcontrol/covid-19-personal-protective-equipment-ppe

[Accessed 19th May 2020]

28. Seto, W. et al. Effectiveness of precautions against droplets and contact in prevention of nosocomial transmission of severe acute respiratory syndrome (SARS). The Lancet 361, 1519-1520 (2003).

29. World Health Organisation. Coronavirus disease (COVID-19) advice for the public. Available from: https://www.who.int/emergencies/diseases/novelcoronavirus-2019/advice-for-public [Accessed 19th May 2020]

30. World Health Organisation. WHO Director-General's opening remarks at the media briefing on COVID-19 - 16 March 2020. Available from: https://www.who.int/dg/speeches/detail/who-directorgeneral-s-opening-remarks-at-the-media-briefing-oncovid-19---16-march-2020 [Accessed 19th May 2020] 31. Zumla, A., Chan, J. F. W., Azhar, E. I., Hui, D. S. C. \& Yuen, K.-Y. Coronaviruses - drug discovery and therapeutic options. Nat. Rev. Drug Discov. 15, 327-347 (2016).

32. Yuen, K.-S., Ye, Z.-W., Fung, S.-Y., Chan, C.-P. \& Jin, D.Y. SARS-CoV-2 and COVID-19: The most important research questions. Cell Biosci. 10, (2020).

33. Mehta, P. et al. COVID-19: consider cytokine storm syndromes and immunosuppression. The Lancet 395, 1033-1034 (2020).

34. Matthay, M. A., Aldrich, J. M. \& Gotts, J. E. Treatment for severe acute respiratory distress syndrome from COVID-19. Lancet Respir. Med. 0, (2020).

35. Ni, Y.-N., Chen, G., Sun, J., Liang, B.-M. \& Liang, Z.-A. The effect of corticosteroids on mortality of patients with influenza pneumonia: a systematic review and metaanalysis. Crit. Care Lond. Engl. 23, 99 (2019).

36. Woodhead, M. Inhaled Corticosteroids Cause Pneumonia ...or Do They? Am. J. Respir. Crit. Care Med. 176, 111-112 (2007).

37. Kim, M. H. et al. Inhaled Corticosteroids in Asthma and the Risk of Pneumonia. Allergy Asthma Immunol. Res. 11, 795-805 (2019).

38. Molina, R. M. de et al. Inhaled corticosteroids and influenza A (H1N1) viral pneumonia. Eur. Respir. J. 40, (2012).

39. ICNARC - $\quad$ Latest news. https://www.icnarc.org/About/Latest-

News/2020/04/04/Report-On-2249-Patients-Critically-IIIWith-Covid-19. 